\title{
La « révolution graphique » d'E.D. Polivanov : le modèle de l'Ouzbékistan
}

\section{Elena Simonato}

\section{(2) OpenEdition}

\section{Journals}

Édition électronique

URL : http://journals.openedition.org/edl/319

DOI : $10.4000 /$ edl.319

ISSN : 2296-5084

\section{Éditeur}

Université de Lausanne

\section{Édition imprimée}

Date de publication : 15 décembre 2009

Pagination : 139-150

ISBN : 978-2-940331-21-5

ISSN : 0014-2026

\section{Référence électronique}

Elena Simonato, « La « révolution graphique » d'E.D. Polivanov : le modèle de l'Ouzbékistan », Études de lettres [En ligne], 4 | 2009, mis en ligne le 15 décembre 2012, consulté le 19 décembre 2020. URL:

http://journals.openedition.org/edl/319; DOI : https://doi.org/10.4000/edl.319 


\section{LA «RÉVOLUTION GRAPHIQUE» D’E.D. POLIVANOV: LE MODÈLE DE L'OUZBÉKISTAN}

Nous nous proposons, dans cette intervention, de mettre en lumière l'apport d'E. Polivanov à la réforme de l'alphabet et de l'orthographe de l'ouzbek dans les années 1920. On a souvent assimilé les réformes des alphabets en Asie centrale à des «révolutions graphiques». Nous montrerons que l'on peut aussi parler de "révolutions» en linguistique puisqu'elles ont amené Polivanov à repenser les problèmes fondamentaux de la linguistique de son temps: la relation entre langue et dialecte, phonétique et orthographe, langue écrite et langue parlée.

\section{Introduction}

La Révolution de 1917 renverse les dogmes, elle met au défi les théories, elle fait tomber les héros d'hier de leur piédestal. Dans le domaine linguistique, elle bouscule aussi bien la situation linguistique que le discours sur la langue; cela a été le cas de l'Union soviétique des années 1920. Parmi tous les débats qui agitent cette époque et qui sont susceptibles de présenter un intérêt pour un historien-linguiste, celui qui tourne autour des réformes d'alphabets pour les langues turkes est un des plus passionnants. L'exemple de la réforme de l'alphabet ouzbek, entreprise entre 1923 et 1930 dans le cadre de l'«édification linguistique», nous servira de point de départ pour une étude d'épistémologie linguistique. Nous verrons alors comment ce projet de réforme amène des linguistes soviétiques, et en premier lieu Evgenij Dmitrievič Polivanov (1891-1938), à repenser les problèmes fondamentaux de la linguistique de leur temps: 
la relation entre langue et dialecte, phonétique et orthographe, langue écrite et langue parlée.

\section{La "révolution graphique» en Ouzbékistan}

\subsection{L'activité scientifique de Polivanov en Asie centrale}

Dès le début des années 1920, plusieurs institutions scientifiques de Petrograd et de Moscou s'engagent dans l'«édification linguistique», ce qui inclut l'élaboration d'alphabets pour les langues non écrites, le passage à l'alphabet latin des langues qui avaient des alphabets à base arabe et l'élaboration de langues standardisées.

E.D. Polivanov peut à juste titre être considéré comme le théoricien principal de la latinisation des alphabets en Asie centrale. Spécialiste de phonétique, des langues turkes et de théorie linguistique au sens le plus large du terme et membre du VCKNTA (Comité central fédéral pour le nouvel alphabet turk) depuis 1928, il contribue également à l'élaboration de nouveaux alphabets pour les peuples turks. Il s'est fait une réputation d'adversaire de la théorie marriste ${ }^{1}$, dans la seconde partie des années 1920 , et il a souffert de répressions ${ }^{2}$.

Polivanov adhère avec ferveur à l'élaboration des alphabets. La citation suivante montre bien son engagement personnel pour cette cause:

Le VCKNTA est en train d'accomplir un acte jamais vu dans l'histoire: il essaye de diriger le processus spontané de la création de l'écriture. Ceci n'était encore jamais arrivé. Bien entendu, en Occident tout comme chez nous, il y aura des scientifiques, des philologues qui diront que c'est impossible, que c'est une tentative absurde. Mais la révolution nous a appris que l'impossible devient possible, et ce qui est possible doit se réaliser ${ }^{3}$.

I. Nikolaj Jakovlevič Marr (1864-1934) est une figure controversée de la linguistique soviétique. Ses études des langues dites "japhétiques", avec l'idée générale d'une évolution des langues par stades, marquent la période des années 1930 où elles deviennent pratiquement la seule direction officielle de recherches.

2. Nous ne nous arrêtons que très brièvement sur cette partie de sa biographie qui a été analysée par V.G. Larcev, E.D. Polivanov, p. 47-50.

3. E.D. Polivanov, in Stenografičeskij otčet Pervogo Plenuma Vsesojuznogo Central'nogo Komiteta Novogo Tjurkskogo Alfavita, p. 79. 
Dès le début des années 1920, Polivanov occupe plusieurs postes à responsabilités au Turkestan (province divisée par la suite en plusieurs formations autonomes). Il est tour à tour nommé adjoint du directeur du Conseil scientifique du Commissariat du peuple pour l'instruction publique de la République du Turkestan puis, en 1923, responsable d'une commission ethnographique pour la réalisation d'un «recensement linguistique " ${ }^{4}$ en vue de la délimitation de l'Asie centrale. C'est au Turkestan également que se déroule une période florissante de son activité d'enseignement et de ses recherches scientifiques.

Dans ses engagements successifs, Polivanov construit une œuvre où il expose aux scientifiques différents projets de réformes d'alphabets et d'orthographe pour l'ouzbek, le kirghiz et le karakalpak, entre autres. Il fait également entendre sa voix dans des revues destinées au large public pour faire valoir ses arguments en faveur du passage à l'alphabet à base latine et de type phonologique. Ses principaux détracteurs représentent l'intelligentsia locale traditionnelle et les maîtres des écoles de Ferghana et de Samarkand, adeptes d'un projet de réforme différent dans son principe (un alphabet à base arabe).

\subsection{Les enjeux de la réforme de l'écriture en Ouzbékistan}

Les problèmes de l'écriture ouzbèke sont débattus dans les congrès du VCKNTA $(1928,1929,1930)$. Malheureusement, les nombreuses rencontres successives ne permettent pas de résoudre toute une série de problèmes abordés.

Il faut savoir qu'à l'époque, élaborer un alphabet pour la langue ouzbèke représentait un défi de taille au vu de la complexité de sa situation dialectologique. Certains éléments du problème sont les suivants:

- le continuum dialectal de l'ouzbek était représenté par deux types de parlers : ceux à synharmonisme (dits aussi "à harmonie des voyelles", avec plus de dix voyelles), principalement répandus dans les villages (ou kichlaks); de l'autre côté, des parlers sans synharmonisme, ceux des villes, avec six voyelles;

- en plus de ces dialectes sans forme écrite, il existait une langue littéraire, employée par les intellectuels et possédant de nombreuses formes

4. Enregistrement de nombreux dialectes centre-asiatiques, principalement iraniens, à Tachkent, Samarkand, dans la vallée du Ferghana. 
archaïques. Les leaders culturels locaux, occupés par la lutte politique, ne pouvaient pas décider quelle devait être la base de l'ouzbek standard. Or, sans avoir résolu ce problème, il était en principe impossible d'élaborer l'alphabet et les bases de l'orthographe.

\section{L'engagement de Polivanov}

\subsection{Polivanov comme linguiste de terrain}

C'est dès 1923 que Polivanov collabore à la description des langues et dialectes parlés en Asie centrale, sur mandat du gouvernement du Turkestan. Ses articles écrits entre 1923 et 1929 portent sur le problème de différentiation entre langue et dialecte, le choix d'une langue «littéraire»: "La composition sonore du dialecte de Tachkent" ["Zvukovoj sostav taškentskogo dialekta»] (1922), Le problème de la latinisation des écritures turkes de l'URSS [Problema latinizacii tureckix pis'mennostej SSSR] (1923), "La nouvelle orthographe kazakh-kirgkize (orthographe de Bajtursun) » " Novaja kazak-kirgizskaja (Bajtursunovskaja) orfografija»] (1924), "Un projet de graphie ouzbèke latinisée » [ Proekt latinskogo šrifta uzbekskoj pis'mennosti»] (1924), Introduction à l'étude de la langue ouzbèke [Vvedenie v izučenie uzbekskogo jazyka] (1925), "A propos de la nouvelle orthographe ouzbèke» [ $\mathrm{O}$ novoj uzbekskoj orfografii »] (1926), "Quelques projets de latinisation des écritures turkes de l'URSS » ["Proekty latinizacii tureckix pis'mennostej SSSR»] (1926), "Quelques exemples des parlers non-iranisés (synharmoniques) de l'ouzbek» " "Obrazcy ne-iranizirovannyx (singarmonističeskix) govorov uzbekskogo jazyka»] (1927). On y trouve des développements qui concernent les rapports cruciaux entre le choix de l'alphabet et l'orthographe, ainsi que des éléments cruciaux au sujet de l'orthographe et du choix de l'alphabet.

Polivanov étudie en détail les propositions des adeptes de la réforme de l'alphabet, qui militent pour un alphabet à base arabe. Il se rend vite compte de la persistance de nombreux clichés et idées reçues qui ne reposent pas sur des recherches de terrain. Il s'emploie donc à démêler le nœud gordien des parlers ouzbeks à partir de ses propres recherches dialectologiques et historiques, secondé en partie par son collègue Konstantin Kuz'mič Judaxin (1890-1975). Polivanov exposera plus tard 
les résultats de cette recherche dialectologique dans le livre Dialectologie ouzbèke et langue littéraire ouzbèke [Uzbekskaja dialektologija i uzbekskij literatutnyj jazyk], en 1933.

\subsection{La situation sociolinguistique des dialectes ouzbeks}

En 1926, dans son article "A propos de la nouvelle orthographe ouzbèke", Polivanov tire une conclusion fondamentale sur les dissemblances entre les différents parlers ouzbeks:

Le fait est que les parlers ouzbeks diffèrent non seulement du point de vue phonétique mais aussi morphologique et, fort considérablement, dans leur vocabulaire (notamment le dialecte ouzbek septentrional et le dialecte de Boukhara) ${ }^{5}$.

Notre chercheur distingue dans le continuum ouzbek trois groupes dialectaux se rattachant "génétiquement» à trois groupes de langues turkes, à savoir :

a) le groupe Sud-Est, dit «tchaghataï»;

b) le groupe Sud-Ouest, dit «oghuz»;

c) le groupe Nord-Ouest, dit «kiptchak».

Les tribus les plus anciennes, les Tchaghataï se sont mélangées avec la population iranophone, suite à quoi leur dialecte a subi une grande influence du persan ${ }^{6}:$ par exemple, la plupart des parlers composant ce dialecte ont perdu le synharmonisme ${ }^{7}$.

Dans l'ensemble, les dialectes se présenteraient comme un «spectre de variations" allant de "fortement iranisés» (ceux de Samarkand et de Boukhara) à "peu iranisés» (celui de Tachkent) et "presque pas iranisés» (les parlers septentrionaux). Polivanov qualifie ces derniers de «purement

5. E.D. Polivanov, «O novoj uzbekskoj orfografii», p. 67.

6. Polivanov emploie à ce sujet le terme de «dialecte hybridisé». - E. S.

7. Le synharmonisme est défini par Polivanov comme un système d'alternances de voyelles - et en partie de consonnes - dans les suffixes, fondé sur la division fondamentale de ces sons selon deux catégories opposées et qui entraîne une division des bases des mots en deux catégories ("d'avant» et «d'arrière»). Il propose d'appeler "synharmonique» un système langagier possédant les suffixes doublets (suffixes verbaux et substantivaux) tels que -lar/-ler, -maq/mak; cf. E.D. Polivanov, Uzbekskaja dialektologija, p. 12 sq. 
turks", car ils ne portent presque pas l'empreinte de l'influence iranienne dans leur système phonologique.

"Le poids sociolinguistique des parlers ouzbeks est proportionnel à leur ancienneté» ${ }^{8}$, constate Polivanov. Ainsi, les parlers du groupe tchaghataï sont surtout répandus dans les villes, les parlers oghuz dans les petites villes et les derniers, dans les villages. Sa thèse est donc la suivante:

Ce sont précisément ces parlers des villes, sans synharmonisme, qui sont appelés à jouer un rôle fondamental dans la constitution de la future langue standardisée ${ }^{9}$.

\subsection{La polémique avec les intellectuels locaux}

Polivanov étudie toutes les étapes de l'élaboration de l'alphabet ouzbek et de l'orthographe. On apprend ainsi qu'en 1922 déjà, la nécessité d'une réforme est soulevée lors d'une séance de la Commission scientifique du conseil d'Etat pour la science du Turkestan. L'année suivante, au printemps 1923, une autre séance réunit aussi bien les promoteurs de la réforme que ses opposants. Enfin, en automne 1923, siège à Boukhara la séance des représentants des organes de l'instruction publique des républiques de l'Asie centrale, date que Polivanov retient comme le début symbolique de la grande transformation orthographique: "Depuis, aucun livre ouzbek ne peut être imprimé selon l'ancien système de l'écriture " ${ }^{10}$, relate-t-il.

Dans un autre texte, Polivanov revient sur la position des intellectuels locaux. Selon lui, ceux-ci étant opposés aux réformes de l'écriture, ils n'ont qu'une vision brouillée de la situation linguistique. En effet, ces intellectuels proposent de garder comme langue écrite en Ouzbékistan le tchaghataï, langue archaïque utilisée en (réservée pour la) littérature; ce qui est une erreur grossière pour Polivanov, puisqu'en cela ils ne font pas de différence autre que statutaire entre le tchaghataï et leurs langues ("dialectes») turkes qui, pour eux, ne sont que des variétés de ce qu'ils désignent par "türki», leur présumée langue commune. D’après lui, leur

8. ED. Polivanov, Vvedenie v izučenie uzbekskogo jazyka, p. 6.

9. E.D. Polivanov, Uzbekskaja dialektologija i uzbekskij literaturnyj jazyk, p. 38.

Io. E.D. Polivanov, «O novoj uzbekskoj orfografii», p. 54. 
vision est fondée sur une mauvaise connaissance de leur propre langue (le dialecte du groupe tchaghataï) qu'ils considèrent comme "purement turke", et en laquelle ils voient la base pour une future koinè - ce qui ne correspond aucunement à la réalité. Notons ici que Polivanov nuance que ce n'est pas volontaire de la part de ces "pro-tchaghataïstes»: ils n'essaient pas de falsifier les faits, ils ne les connaissent pas. Ainsi, par exemple, se représentent-ils tous les dialectes ouzbeks comme gardant l'harmonie vocalique, qui pour eux rime avec la "pureté» de la langue ${ }^{11}$.

Ces intellectuels, continue Polivanov, n'estiment digne d'étude et d'emploi littéraire que le tchaghataï, du fait de son prestige. "Mais comment, se demande-t-il, ces parlers, plus iranisés que les autres, peuvent-ils être compris et sentis comme "purement turks" par leurs voisins turkophones" ${ }^{12}$, sinon comme continuateurs du tchaghataï écrit, aussi incompréhensible soit-il? En réalité, répond-il, le tchaghataï n'est qu'une variété écrite du dialecte de Ferghana ${ }^{13}$, c'est-à-dire pas du tout une koinè. A cela s'ajoute que, suite à une attitude puriste envers les textes, le tchaghataï a fini par se différencier fortement de la langue parlée des villes, en conservant des formes disparues depuis longtemps. Il est donc devenu incompréhensible non seulement pour le peuple, mais aussi pour la nouvelle intelligentsia des villes. Le tchaghataï ne peut donc aucunement correspondre aux exigences primordiales du VCKNTA: se fonder sur un dialecte compréhensible pour tous.

La proposition de Polivanov est donc de retenir, comme base de l'ouzbek standardisé, le dialecte de Tachkent, et ceci pour plusieurs raisons. Ses nombreuses études historiques sur différentes langues ont démontré, premièrement, que la langue (ou le dialecte) de la ville, d'une capitale de surcroît, devient toujours le dialecte de prestige; deuxièmement, que les autres dialectes ouzbeks perdent progressivement le synharmonisme.

II. E.D. Polivanov, Uzbekskaja dialektologija i uzbekskij literaturnyj jazyk, p. 38 sq.

I2. C'est le cas des habitants de Khorezme et ceux de l'Ouzbékistan central.

13. En effet, les recherches de Polivanov et de Judaxin démentent donc l'opinion selon laquelle le tchaghataï est une koinè vivante et donc utilisable comme base d'une future koinè écrite. 


\section{Les choix stratégiques de Polivanov}

\subsection{Polivanov réformateur de l'orthographe}

La discussion sur la réforme de l'orthographe ouzbèke, qui concerne l'expression graphique du synharmonisme et donc le choix du dialecte de référence, doit être resituée dans son contexte. En effet, ce qui est en jeu ici, ce n'est pas la forme graphique de l'alphabet - arabe ou latine - mais bien son principe linguistique.

Dans tous les débats qui touchent aux principes linguistiques des nouveaux alphabets à base latine, Polivanov se prononce pour une écriture à base phonétique ${ }^{14}$ pour toutes les langues concernées par la latinisation, principe défendu en chœur par les deux autres linguistes les plus renommés du moment, Nikolaj Feofanovič Jakovlev (1892-1974) et Lev Vladimirovič Ščerba (1880-1944), au Premier Congrès Turkologique en 1926. Mais dans le cas de l'alphabet ouzbek, ce principe est difficile à réaliser au vu de la grande variation dialectale, il est inadapté pour les dialectes à synharmonisme :

On ne peut pas écrire phonétiquement (or, c'est dans la possibilité d'écrire phonétiquement que réside tout l'avantage pédagogique d'une graphie rationalisée) le nouvel alphabet ouzbek en un dialecte possédant le synharmonisme ${ }^{15}$.

Polivanov propose, pour l'alphabet et l'orthographe ouzbeks, une solution qui pourrait à juste titre être considérée comme révolutionnaire. D'après lui, il serait vain d'essayer de refléter le phénomène de synharmonisme dans l'orthographe. Il insiste également sur la nécessité de se fonder, dans l'élaboration de l'orthographe, sur le dialecte de Tachkent.

Nous citons un exemple particulièrement éloquent des difficultés auxquelles étaient confrontés les auteurs du projet de l'orthographe ouzbèke et de la solution proposée par Polivanov:

I4. Le principe que Polivanov suggère de suivre consiste à faire correspondre à chaque graphème ce qu'il appelle une "représentation sonore ", c'est-à-dire, un phonème dans la terminologie de l'école phonologique de Leningrad dont il était adepte. Nous dirions aujourd'hui "représentation phonologique».

I5. E.D. Polivanov, "O novoj uzbekskoj orfografii», p. 63. 
Prenons un exemple. Dans le dialecte de Tachkent (qui ne connaît pas la distinction des phonèmes o et $\ddot{o}$, mais un seul phonème $\mathrm{o}$, les mots «feu» - [ot], «herbe» - [ot], et "passe» (impératif) [ot] - sonnent de manière identique: et, par la suite, s'écrivent de manière identique. Autrement on ne peut pas les représenter avec la nouvelle graphie. Mais cette manière d'écrire ces mots est juste uniquement pour le dialecte de Tachkent (et des parlers qui lui sont proches, c'est-à-dire des parlers iranisés). Dans un dialecte qui a le synharmonisme, comme le dialecte ouzbek septentrional, ces mots sonnent différemment: "feu» - [od] (ou plutôt [o:d], avec un [o] long), «herbe» - [ot], et "passe» [öt]. Il n'est pas possible de transmettre les différences des voyelles dans ces mots avec la graphie actuelle, qui ne possède que six lettres pour les voyelles ${ }^{16}$.

Polivanov entre alors en polémique avec ses opposants en attirant l'attention sur le fait suivant:

Certains adeptes de la réforme graphique recommandent en effet: «Ecris en respectant le synharmonisme, même si ta langue ne le possède pas». La morphologie, relativement simple, du parler de Tachkent devient trop compliquée écrite de cette façon, et un locuteur de ce parler doit, pour pouvoir écrire un mot, réfléchir comment ce mot devrait être prononcé dans les parlers qui ont le synharmonisme, ou aller demander des informations à Khiva ou au Kazakhstan ${ }^{17}$.

\subsection{Polivanov penseur du futur linguistique}

Le point fort de l'option proposée par Polivanov (choisir comme base de l'orthographe un dialecte sans synharmonisme) repose sur ses conclusions d'ordre sociolinguistique. Rappelons que Polivanov appelait non seulement à étudier le passé des langues, mais également à faire des pronostics sur leur futur: d'après lui, dans les dialectes qui le possèdent encore, le synharmonisme ne pourrait que se perdre, sous l'influence du dialecte standard (de Tachkent) et du mélange des langues. Ses

I6. Ibid., p. 63 sq.

17. Ibid., p. 65. Polivanov mentionne ici le Kazakhstan parce que la région de Tchimkent, où réside une partie des ouzbékophones, faisait alors partie du Kazakhstan. 
objections sont dirigées contre l'essentiel de la méthode de ses opposants qui cherchent à figer l'évolution de la langue parlée:

On pourrait de premier abord croire que chez les promoteurs de la réforme ouzbèke qui exigent que tout le monde écrive selon les règles de l'harmonie des voyelles, il y a une volonté importante, volonté qui nous amènera à la création d'une langue littéraire ouzbèke unie. Mais en réalité, on a affaire ici à une mauvaise connaissance de l'évolution de la langue et de la société, et à une tentative qui emploie de faux moyens en fin de compte ${ }^{18}$.

Polivanov appelle également à ne pas se précipiter pour créer une «langue ouzbèke unie». Il est même établi qu'il n'exclut pas la création de deux ou trois langues ouzbèkes, sur la base des trois dialectes susmentionnés ${ }^{19}$. Pour lui, le plus important dans un premier temps est de doter la langue ouzbèke d'une orthographe et d'un alphabet. Tous les locuteurs de l'ouzbek pourront ainsi communiquer par écrit, ce qui permettra également l'essor de l'alphabétisme et de la littérature en cette langue. Une réforme ultérieure pourrait être réalisée sur la base de l'évolution sociolinguistique et dialectale future, suivie de près par les linguistes:

Laissons apparaître dans l'écriture toute la richesse des dialectes de la parole ouzbèke. Au fur et à mesure que l'unification nationale et culturelle des Ouzbeks exigera un moyen de communication entre eux, c'est-à-dire une langue standard, un parler l'emportera sur les autres (ou probablement deux) ; l'écriture se fixera alors sur ce parler-là (cela peut être un seul parler ou deux, l'un avec synharmonisme, et l'autre sans) ${ }^{20}$.

\section{Conclusion}

Dans ses publications, Polivanov a maintes fois souligné le lien entre la révolution dans la société soviétique, dans les esprits et en linguistique. $\mathrm{Vu}$ de près, ce lien se révèle être bien plus qu'un parallèle symbolique. Dans le cas que nous avons étudié dans cet article, la réforme

I8. Ibid., p. 66.

I9. Ibid., p. 41 et 44 .

2o. Ibid., p. 67. 
de l'alphabet et de l'orthographe ouzbeks, Polivanov a su opérer une véritable révolution, un changement de perspective dans la manière de voir la situation dialectologique en Ouzbékistan, ceci à partir des trois points forts de sa conception : l'approche sociolinguistique, le pronostic du futur linguistique et le rôle fédérateur de l'écriture dans la constitution d'une langue standardisée.

En cela nous pouvons souligner la modernité de sa pensée. En effet, des positions théoriques ont eu des effets décisifs sur les choix et les résultats de ses successeurs, puisqu'à sa suite, entre autres, les dimensions méthodologiques et théoriques ont été étroitement articulées dans l'élaboration des alphabets.

Elena Simonato Université de Lausanne 


\section{BIBLIOGRAPHIE}

Larcev, Vasilij Grigor'evič, E.D. Polivanov: stranicy žizni i dejatel'nosti, Moskva, Nauka, 1988.

Polivanov, Evgenij Dmitrievič, "Zvukovoj sostav taškentskogo dialekta", Nauka i prosveščnie, 1 (1922), p. 17-19.

—, Problema latinizacii tureckix pis'mennostej SSSR, Moskva, Institut vostokovedenija, 1923.

—, Novaja kazak-kirgizskaja (Bajtursunovskaja) orfografija, Taškent, Turkpečat', 1924.

—, "Proekt latinskogo šrifta uzbekskoj pis'mennosti», Bjulleten' 1-go Sredne-Aziatskogo gosudarstvennogo universiteta, 6 (1924), p. 158-159.

—, Vvedenie v izučenie uzbekskogo jazyka, Taškent, Turkpečat', 1925.

—, "O novoj uzbekskoj orfografii », in E.D. Polivanov, Kratkaja grammatika uzbekskogo jazyka, Taškent/Moskva, Turkpečat', 1926, partie 2, p. 54-89.

—, Proekty latinizacii tureckix pis'mennostej SSSR. K Tjurkologičeskomu s"ezdu II, Taškent, 1926, cité in E.D. Polivanov, Selected Works, Articles on General Linguistics, The Hague/Paris, Mouton, 1974, p. 38-39.

—, "Obrazcy ne-iranizirovannyx (singarmonističeskix) govorov uzbekskogo jazyka ", Izvestija Akademii Nauk SSSR, otdelenie gumanitarnyx nauk, 1 (1927), p. 511-537.

—, Uzbekskaja dialektologija i uzbekskij literaturnyj jazyk, Taškent, Uzgosizdat, 1933.

Stenografičeskij otčet Pervogo Plenuma Vsesojuznogo Central'nogo Komiteta Novogo Tjurkskogo Alfavita, zasedavšego v Baku ot 3-go po 7 ijunja 1927 goda, Moskva, VCKNTA, 1927. 